\title{
The CEACAM1 tumor suppressor is an ATM and p53-regulated gene required for the induction of cellular senescence
} by DNA damage

\author{
A-P Sappino ${ }^{1}$, R Buser ${ }^{2}$, Q Seguin ${ }^{3}$, M Fernet ${ }^{4}$, L Lesne ${ }^{2}$, F Gumy-Pause ${ }^{2}$, W Reith ${ }^{3}$, V Favaudon ${ }^{4}$ and SJ Mandriota ${ }^{2}$
}

The p53 tumor-suppressor protein has a key role in the induction of cellular senescence, an important barrier to cancer development. However, very little is known about the physiological mediators of cellular senescence induced by p53. CEACAM1 is an immunoglobulin superfamily member whose expression is frequently lost in human tumors and exhibits tumor-suppressor features in several experimental systems, including Ceacam 1 knockout mice. There is currently little understanding of the pathways and mechanisms by which CEACAM1 exerts its tumor-suppressor function. Here we report that CEACAM1 is strongly upregulated during the cellular response to DNA double-strand breaks (DSBs) starting from the lowest doses of DSB inducers used, and that upregulation is mediated by the ataxia telangiectasia mutated (ATM)/p53 pathway. Stable silencing of CEACAM1 showed that CEACAM1 is required for p53-mediated cellular senescence, but not initial cell growth arrest, in response to DNA damage. These findings identify CEACAM1 as a key component of the ATM/p53-mediated cellular response to DNA damage, and as a tumor suppressor mediating cellular senescence downstream of p53.

Oncogenesis (2012) 1, e7; doi:10.1038/oncsis.2012.7; published online 16 April 2012

Subject Category: tumour suppression

Keywords: CEACAM1; ATM; p53; DNA damage; cellular senescence

\section{INTRODUCTION}

CEACAM1 is an immunoglobulin superfamily member that regulates several cellular functions-including angiogenesis, insulin metabolism and T-cell function-and has features of a tumor suppressor in several experimental systems. CEACAM1 is expressed in epithelial cells, leukocytes, T cells and endothelial cells. It exists as a number of splice variants with most isoforms harboring a transmembrane domain and either a long or a short cytoplasmic domain. The short isoforms encode 10 cytoplasmic residues lacking phosphorylation sites. In contrast, the long isoforms encode approximately 70 cytoplasmic amino acids, including several serine, threonine and tyrosine residues that can be phosphorylated and participate in signal transduction. This domain is required for CEACAM1's tumor-suppressor function. Both isoforms are co-expressed in most CEACAM1-expressing cells. In several human tumor types-including colon, prostate, endometrium and breast tumors-expression of CEACAM1 is frequently reduced or completely lost. Additional evidence for a CEACAM1 tumor-suppressor function is the reduced in vitro and in vivo growth capacity of tumor cells in which CEACAM1 has been reintroduced and the increased colon tumorigenesis of CEACAM1 knockout mice treated with azoxymethane. ${ }^{1,2}$ Although CEACAM1 is known to act as a general negative regulator of cell proliferation, there is currently little understanding of the mechanisms by which the loss of CEACAM1 contributes to cancer. In particular, there are currently no reports placing CEACAM1 in the ataxia telangiectasia mutated (ATM)/p53 pathway or, more generally, in the DNA damage response, whose integrity is a well-established and key component of tumor suppression.

We previously reported that functional inhibition of ATM by either stable silencing or pharmacological inhibition selectively transforms human mammary epithelial cells in the absence of exogenous DNA damage, whereas in other human cell types ATM inhibition had no effect. ${ }^{3}$ These results provided the first in vitro model of human cell transformation by the loss of function of ATM, and showed that human mammary epithelial cells are particularly sensitive to decrease in ATM activity, which is consistent with the increased breast cancer susceptibility of ATM mutation carriers. ${ }^{4}$ Based on these results, we reasoned that human mammary epithelial cells would be more informative than other cell types for the study of the tumor-suppressor function of ATM. As the ATM-mediated transcriptional responses to DNA damage have key roles in ATM tumor-suppressor function, we studied such responses in human mammary epithelial cells. We found that CEACAM1 is strongly upregulated during the ATMmediated DNA damage response, that this regulatory effect is mediated by $\mathrm{p} 53$ and that induction of CEACAM 1 by DNA damage is required for the establishment of cellular senescence, an important barrier to cancer development. ${ }^{5-7}$

\section{RESULTS}

CEACAM1 was observed to be upregulated during the ATMdependent DNA damage response in complementary DNA (cDNA)

\footnotetext{
${ }^{1}$ Clinique des Grangettes, Geneva, Switzerland; ${ }^{2}$ Hemato-Oncology Unit, Department of Pediatrics, Faculty of Medicine/University Hospital of Geneva, Geneva, Switzerland; ${ }^{3}$ Department of Pathology and Immunology, Faculty of Medicine, Geneva, Switzerland and ${ }^{4}$ Institut Curie, Orsay, France. Correspondence: Dr SJ Mandriota, Hemato-Oncology Unit, Department of Pediatrics, Faculty of Medicine/University Hospital of Geneva, Avenue de la Roseraie 64, Geneva 1205, Switzerland. E-mail: stefano.mandriota@unige.ch Received 13 January 2012; revised 14 February 2012; accepted 15 February 2012
} 
microarray experiments using MCF-10A human mammary gland epithelial cells with or without stable ATM silencing ${ }^{3}$ and neocarzinostatin (NCS) as a DNA double-strand break (DSB) inducer (data not shown). Real-time quantitative PCR and western blotting confirmed this regulatory effect, CEACAM1 being strongly induced at the mRNA and protein levels in MCF-10A cells with normal ATM expression, whereas induction was reduced by approximately $50 \%$ in their counterpart with stable ATM silencing, which retains partial ATM expression (Figures 1a and b). The ATM kinase-specific inhibitor $\mathrm{KU}-55933^{8}$ had a strong inhibitory effect on CEACAM1 induction by NCS at the mRNA and protein levels in the parental MCF-10A cell line (Figure 1C; see also the effect of KU55933 on CEACAM1 mRNA induction by the same NCS concentration $(5.46 \mathrm{nM})$ in Figure 1e). At the concentration used $(10 \mu \mathrm{M}), \mathrm{KU}-55933$ inhibits ATM kinase activity in MCF-10A cells almost completely (Supplementary Figure S1). As assessed in several independent experiments using MCF-10A cells or primary human mammary epithelial cells, CEACAM1 induction by DNA damage was relatively slow when compared with p21/Waf1 or BTG2, which are also known to be induced by DNA damage, with the CEACAM1 mRNA peaking at $6 \mathrm{~h}$ and the protein peaking at $12-24 \mathrm{~h}$ in experiments using NCS or X-rays. Examples of the
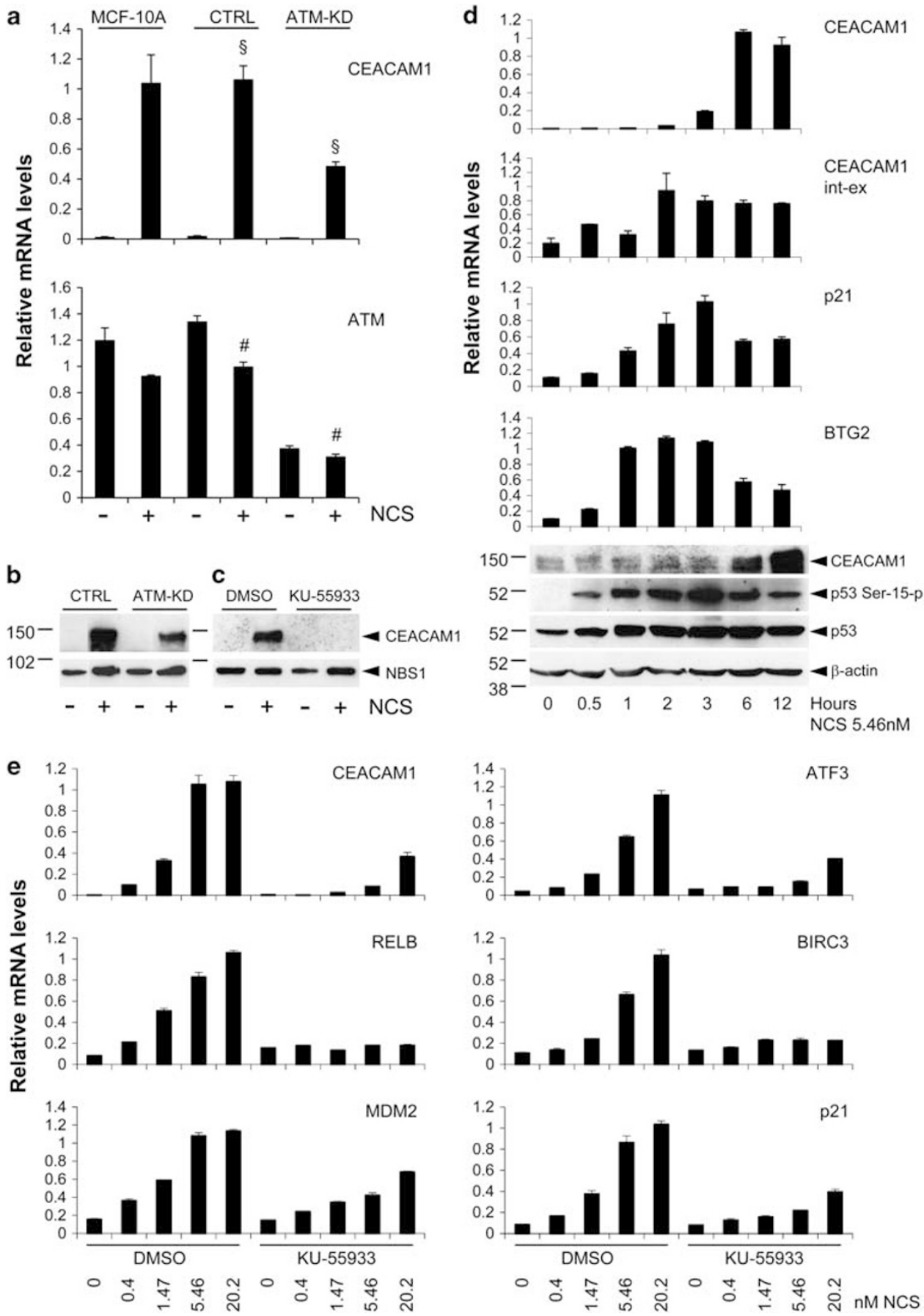
results obtained are shown in Figure 1d and Supplementary Figure S2. The CEACAM1 mRNA peak was preceded by a peak of the CEACAM1 primary transcript (CEACAM1 int-ex, Figure 1d) thus suggesting a transcriptional effect.

In dose-response experiments, CEACAM1 was induced starting from $0.4 \mathrm{~nm}$ NCS, a low dose that increases the number of $\gamma-\mathrm{H} 2 \mathrm{AX}$ nuclear foci/cell by approximately 1.8 -fold and barely promotes ATM autophosphorylation on Ser 1981 (Figure 1e, Supplementary Figures S3 and S4, and data not shown). The dose-response of CEACAM1 was similar to that of other genes known to be regulated by ATM through p53 (MDM2, p21 Waf1, ATF3) or NF- $\mathrm{BB}$ (BIRC3, RELB) (Figure 1e). For all these genes, induction was potently reduced by the KU-55933 inhibitor (Figure 1e). By isoform specific real-time quantitative PCR, MCF-10A cells expressed both the short and long CEACAM1 isoforms, upregulated by NCS to a similar extent (data not shown; see similar results in Supplementary Figure S8 for HCT116 cells). Similar to p21 2 af1 and BTG2, CEACAM1 induction was also observed in response to X-Rays or etoposide, starting from low doses ( $0.3 \mathrm{~Gy}$ or $1 \mu \mathrm{M}$, respectively) (Supplementary Figure S5).

As p53 is a major downstream effector of the ATM-mediated transcriptional response to DNA damage, we next assessed whether CEACAM1 induction by NCS is dependent on p53. A p53 short interfering (si)RNA (p53 siRNA no. 3) that inhibits p53 mRNA and protein expression by approximately $80 \%$ (Figure 2a, Supplementary Figure S6) strongly reduced induction of CEACAM 1 by NCS (Figure 2a). CEACAM1 mRNA induction by NCS was also strongly diminished by the ATM-specific inhibitor KU-55933 (Figure 2a), thus confirming the results obtained with stable ATM silencing (Figures $1 \mathrm{a}$ and b), or with KU-55933 (Figures 1c and e). Of the known p53 target genes analyzed in the same experiment, p21 Waf1 ATF3, BTG2 and MDM2 exhibited a similar regulation profile. In contrast, induction of RELB and BIRC3, which are known to be regulated by ATM through NF- $\kappa B$ but not through $\mathrm{p} 53$, was reduced by the KU-55933 inhibitor but not by the p53 siRNA (Figure 2a and data not shown). This confirmed previous results ${ }^{9,10}$ and demonstrated the specificity of the p53 siRNA. Virtually identical results were obtained in a second experiment using a different p53 siRNA (Supplementary Figures S6, S7). To consolidate the observation that CEACAM1 is a p53-regulated gene, and to explore the possibility that this regulation also occurs in other cell types, we analyzed CEACAM1 regulation by X-Rays or NCS in HCT116 or SW48 human colon cancer cells having wild type or inactivated p53, which are well established models of p53 function. ${ }^{11}$ In these cell lines, similar to known p53 target genes, CEACAM1 mRNA and protein were induced by X-Rays or NCS in the presence, but not in the absence of functional p53 (Figures $2 \mathrm{~b}$ and $\mathrm{d}$; Supplementary Figure S8). Consistent with these results, CEACAM1 was not upregulated by NCS in HaCaT human keratinocytes or MDA-MB-231 human breast cancer cells (data not shown), that carry mutant $\mathrm{p} 53 .{ }^{12,13}$
To further corroborate the regulation of CEACAM1 by p53 we analyzed CEACAM1 regulation by nutlin-3, a small molecule that specifically inhibits the $\mathrm{p} 53 / \mathrm{MDM} 2$ negative regulatory interaction targeting p53 for proteasomal degradation, thus leading to p53 stabilization and activation in the absence of DNA damage. ${ }^{14}$ CEACAM1 was upregulated by nutlin-3 in a time- and dosedependent manner (Figure 2c). p21/Waf1 and BTG2, two wellknown p53 target genes, were also upregulated by nutlin- 3 in these experiments, the difference being that CEACAM1 induction was slightly slower (Figure 2c, left panel). CEACAM1 was also upregulated by NCS or nutlin-3 in SH-N-SH, KCNR and IMR32 human neuroblastoma cells, human lymphocytes and MCF-12A human mammary epithelial cells, all having functional ATM and p53 (data not shown).

Real-time quantitative PCR analysis of CEACAM1 in NCS-treated MCF-10A cells revealed an early increase in the primary transcript (CEACAM1 int-ex; Figure 1d, Supplementary Figure S9), suggesting that p53 upregulates CEACAM1 at the level of transcription. To investigate the possibility that $\mathrm{p} 53$ regulates CEACAM1 expression by binding directly to CEACAM1 regulatory sequences, we performed quantitative p53 chromatin immunoprecipitation (ChIP) experiments for putative p53 binding sites (as assessed by the p53MH algorithm) ${ }^{15}$ and ChIP-seq experiments with NCStreated MCF-10A cells using two different p53 antibodies validated for ChIP (DO-1 or Invitrogen (Zug, Switzerland) cat. no. 491031). Although ChIP-seq and quantitative ChIP experiments suggested that binding of $\mathrm{p} 53$ might be induced at specific regions in the CEACAM1 gene, these binding signals were significantly less evident than at known p53 target genes such as CDKN1A (p2 ${ }^{\text {Waf1 }}$ ) and MDM2 (data not shown). To investigate the possibility that $\mathrm{p} 53$ regulates CEACAM1 indirectly, we analyzed the effect of NCS on the known major transcriptional regulators of CEACAM1, namely IRF1, IRF2, USF1 and USF2 ${ }^{16}$ in MCF-10A cells. NCS $5.46 \mathrm{~nm}$ had little or no effect on the IRF-2, USF1, or USF2 mRNA levels. In contrast, it increased IRF1 mRNA levels by $\sim 2.5-$ fold at $4 \mathrm{~h}$ (Supplementary Figure S10 and data not shown). This increase, however, was dependent on ATM but not on p53 (Supplementary Figure S10). Taken together, our experiments suggested that $\mathrm{p} 53$ regulation of CEACAM1 is indirect but not mediated by IRF1, or that $\mathrm{p} 53$ binding to CEACAM1 regulatory sequences cannot be detected convincingly under the ChIP conditions used.

To investigate the role of CEACAM1 in the DNA damage response, we generated MCF-10A or HCT116 cells with stable CEACAM1 silencing using the small hairpin RNA (shRNA) vectors 025,026 or 028 , targeting both the long and short isoforms of CEACAM1. The same vector coding for a scrambled shRNA (CTRL) was used to generate controls. These cells displayed significantly reduced levels of CEACAM1 at the mRNA and protein levels (Figures $3 a$ and $b$ ). As the activation of cell cycle checkpoints is a

Figure 1. CEACAM1 is upregulated by NCS in an ATM-dependent manner. (a) Parental MCF-10A cells (MCF-10A) or MCF-10A cells stably expressing an ATM shRNA vector (ATM-KD) or a scrambled shRNA vector (CTRL) as a control were incubated for $4 \mathrm{~h}$ in the presence or the absence of $20 \mathrm{nM}$ NCS. At the end of the incubation, total RNA was extracted and analyzed for the levels of CEACAM1 or ATM expression by quantitative real-time PCR. The values in the graph represent the mean \pm s.e.m. from three independent experiments. ${ }^{\S} P<0.005$; ${ }^{\#} P<0.001$. (b) MCF-10A cells stably expressing an ATM shRNA vector (ATM-KD) or a scrambled shRNA (CTRL) were incubated for $16 \mathrm{~h}$ in the presence or the absence of $5.46 \mathrm{~nm}$ NCS. At the end of the incubation, total proteins were extracted and analyzed for the levels of CEACAM1 or Nijmegen breakage syndrome 1 (NBS1) expression by western blotting. (c) Parental MCF-10A cells were incubated for $1 \mathrm{~h}$ in the presence of $10 \mu \mathrm{M}$ KU-55933 or the same dilution of dimethyl sulfoxide (DMSO (solvent)) as a control, followed by $16 \mathrm{~h}$ incubation in the presence or the absence of NCS $5.46 \mathrm{nM}$. At the end of the incubation, total proteins were extracted and analyzed for the levels of CEACAM1 or NBS1 expression by western blotting. In $\mathbf{b}$ and $\mathbf{c}$, numbers on the left indicate $\mathrm{kDa}$. (d) MCF-10A cells were incubated in the presence of $5.46 \mathrm{nM}$ NCS for the times indicated. At the end of the incubation, the cells were split into two parts and either processed for protein extraction or for total RNA purification. RNAs were analyzed for the levels of CEACAM1, CEACAM1 primary transcript (CEACAM1 int-ex), p21/Waf1 or BTG2 by quantitative real-time PCR in triplicates. Error bars indicate s.d. within the internal replicates. Proteins were analyzed for the levels of CEACAM1, p53 Ser $15-p$, p53 or $\beta$-actin (lower panel). Numbers on the left indicate kDa. (e) MCF-10A cells were incubated for $1 \mathrm{~h}$ in the presence of $10 \mu \mathrm{M}$ KU55933 or the same volume of DMSO (solvent) as a control, followed by $4 \mathrm{~h}$ in the presence of the indicated concentrations of NCS. At the end of the incubation total RNAs were purified and analyzed for the levels of the indicated genes by quantitative real-time PCR in triplicates. Error bars indicate s.d. within the internal replicates. 
key component of the DNA damage response, we analyzed the cell cycle in NCS-treated MCF-10A cells with or without stable CEACAM1 silencing. We found no substantial differences up to
$24 \mathrm{~h}$ after NCS treatment. A strong and progressive reduction of cells in $\mathrm{S}$ phase was observed both in controls and stable transfectants with CEACAM1 silencing (Figure $3 c$ ). This result is

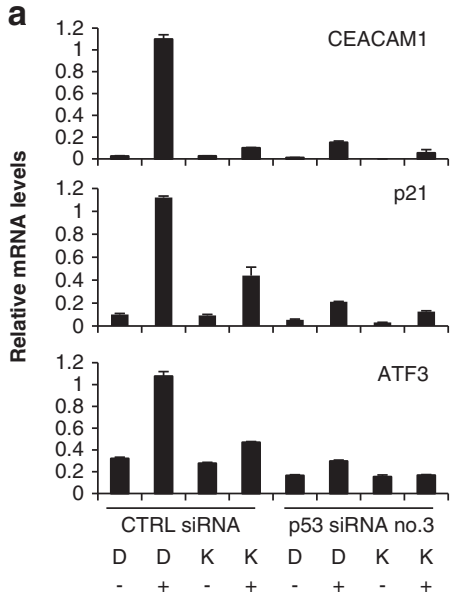

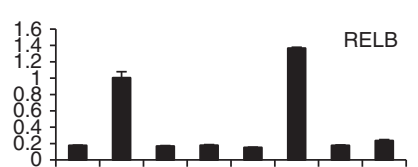

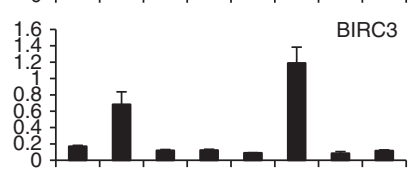

1.4

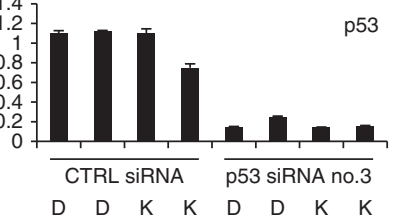

$\begin{array}{llllllll}D & D & K & K & D & D & K & K \\ - & + & - & + & - & + & - & +\end{array}$

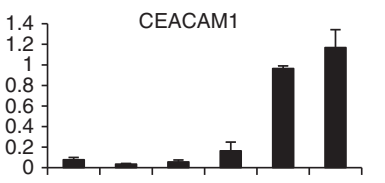

p21

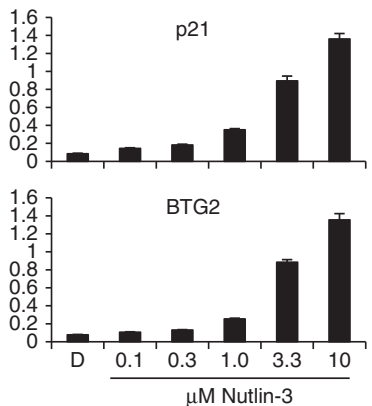

$\mu \mathrm{M}$ Nutlin-3 b
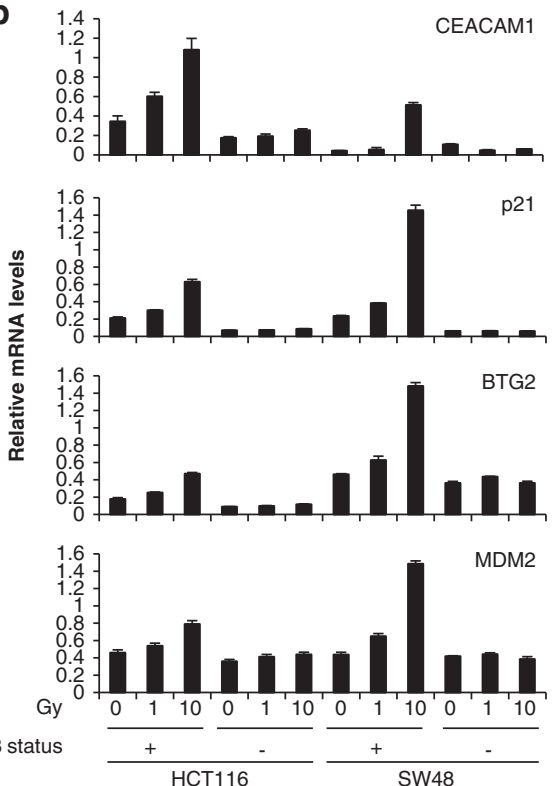

d

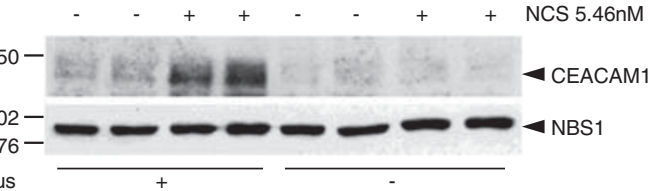

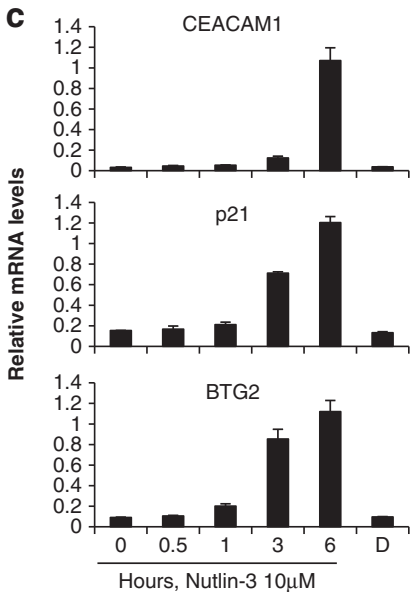

Figure 2. Induction of CEACAM1 by NCS or X-Rays in MCF-10A, HCT116 or SW48 cells is dependent on p53. (a) MCF-10A cells were transfected with p53 siRNA no.3 or a control (CTRL) siRNA as indicated. Two days later, $10 \mu \mathrm{M}$ KU-55933 (K) or dimethyl sulphoxide (DMSO) (D) (solvent) was added. One hour later the cells were incubated in the presence or the absence of $20 \mathrm{~nm}$ NCS as indicated for an additional $4 \mathrm{~h}$. At the end of the incubation total RNAs were purified and analyzed for the levels of the indicated genes by quantitative real-time PCR in triplicates. Error bars indicate s.d. within the internal replicates. (b) HCT116 or SW48 cells with wild type or inactivated p53 were irradiated with the indicated doses of X-rays and incubated at $37^{\circ} \mathrm{C}$ for $6 \mathrm{~h}$. At the end of the incubation total RNAs were purified and analyzed for the levels of the indicated genes by quantitative real-time PCR in triplicates. Error bars indicate s.d. within the internal replicates. (c) MCF-10A cells were incubated for the indicated times in the presence of $10 \mu \mathrm{m}$ nutlin-3, or for $6 \mathrm{~h}$ in the presence of DMSO (D) (solvent) (left) or for $6 \mathrm{~h}$ in the presence of the indicated concentrations of nutlin-3 or the same volume of DMSO (D) (right). At the end of the incubation total RNAs were purified and analyzed for the levels of the indicated genes by quantitative real-time PCR in triplicates. Error bars indicate s.d. within the internal replicates. (d) HCT116 cells with wild type or inactivated p53 were incubated in the presence or the absence of NCS $5.46 \mathrm{nM}$ for $16 \mathrm{~h}$ in duplicate as indicated. At the end of the incubation the cells were lysed and analysed for the levels of CEACAM1 or NBS1 by western blotting.

Figure 3. Establishment and phenotype of MCF-10A cells or HCT116 cells with stable silencing of CEACAM1. (a) HCT116 cells stably transfected with CEACAM1 shRNA vector no. 26 (CEACAM1-KD 026), with CEACAM1 shRNA vector no. 28 (CEACAM1-KD 028) or with a control shRNA vector (CTRL) were lysed and analyzed for CEACAM1 or NBS1 expression levels by western blotting. One of two experiments with similar results is shown. (b) MCF-10A cells stably transfected with CEACAM1 shRNA vector no. 25 (CEACAM1-KD 025), with CEACAM1 shRNA vector no. 26 (CEACAM1-KD 026) or with a control shRNA vector (CTRL) were analyzed for CEACAM1 mRNA levels by quantitative real-time PCR. Error bars indicate s.d.; $n=2$. (c) MCF-10A cells stably transfected with CEACAM1 shRNA vector no. 25 (MCF10A CEACAM1-KD 025) or with a COntrol shRNA vector (MCF10A ${ }^{C T R L}$ ) were incubated in the presence or the absence of NCS $1.47 \mathrm{nM}$ for the indicated time points, labeled with BrdU (10 $\mu \mathrm{M}$, $20 \mathrm{~min}$ ), fixed with ice-cold ethanol and treated for analysis of the cell cycle phase distribution by flow cytometry using a FACSCalibur apparatus (Becton-Dickinson Biosciences, Le Pont-De-Claix Cedex, France). At least $10^{4}$ events were recorded and data analysis was done with CellQuest Pro software (Becton-Dickinson Biosciences). (d) MCF-10A cells stably transfected with CEACAM1 shRNA vector no. 25 (CEACAM1-KD 025), with CEACAM1 shRNA vector no. 26 (CEACAM1-KD 026) or with a control shRNA vector (CTRL) were seeded in $60 \mathrm{~mm}$ Petri dishes at the density of 1500 cells/dish. On the following day the cells were treated with the indicated dose of etoposide for $1 \mathrm{~h}$, washed with phosphate-buffered saline, and allowed to grow in new medium at $37^{\circ} \mathrm{C}$. After 7 days, the colonies were fixed with methanol, stained with crystal violet, and counted. 
consistent with the relatively late induction of CEACAM1 by DNA damage, compared with p21/Waf1 (Figure 1d). To investigate the possibility that CEACAM1 has a role at a later stage in the DNA damage response, we performed proliferation assays where MCF-
10A stable transfectants with or without stable CEACAM1 silencing (1500 cells per $60-\mathrm{mm}$ Petri dish) were allowed to grow for 7 days after NCS or etoposide treatment. Seven days after treatment, a higher number of colonies was observed in MCF-10A CEACAM1-KD 025

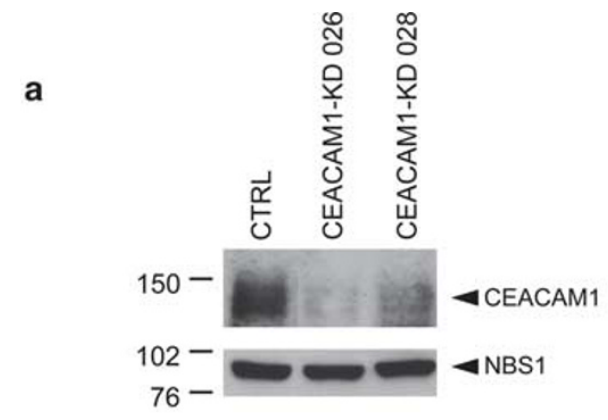

b

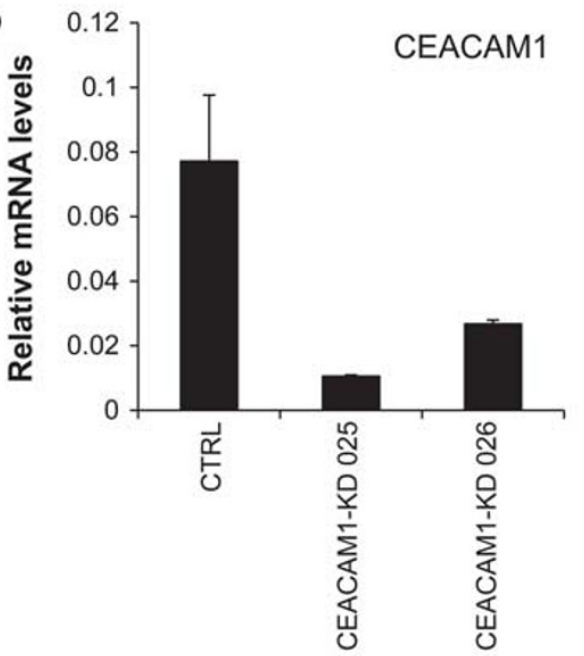

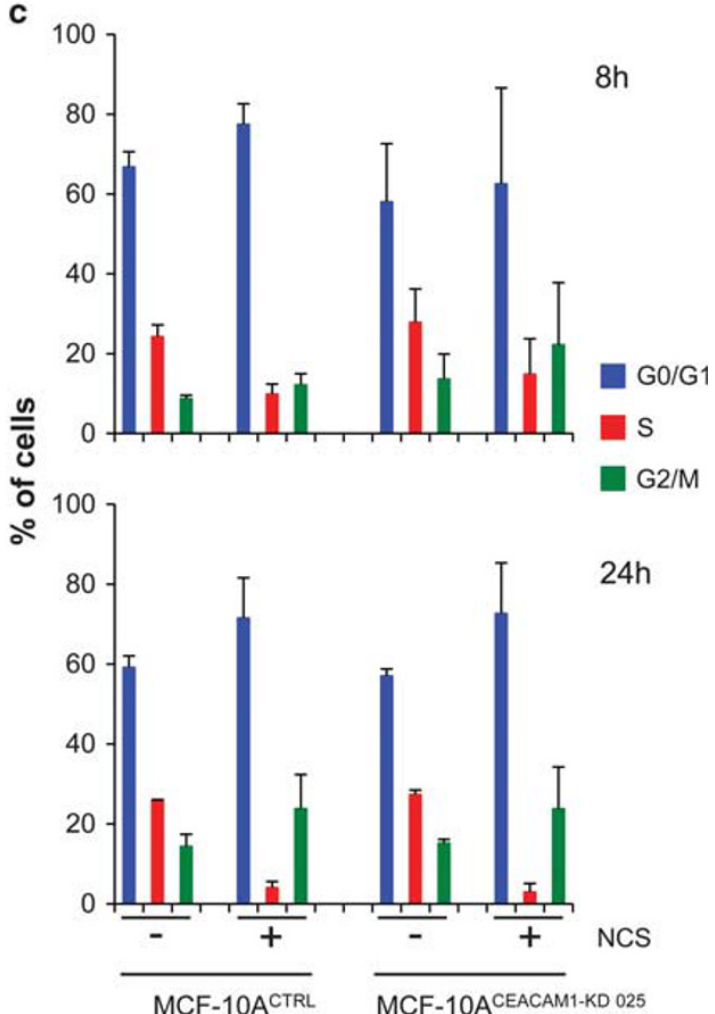

d

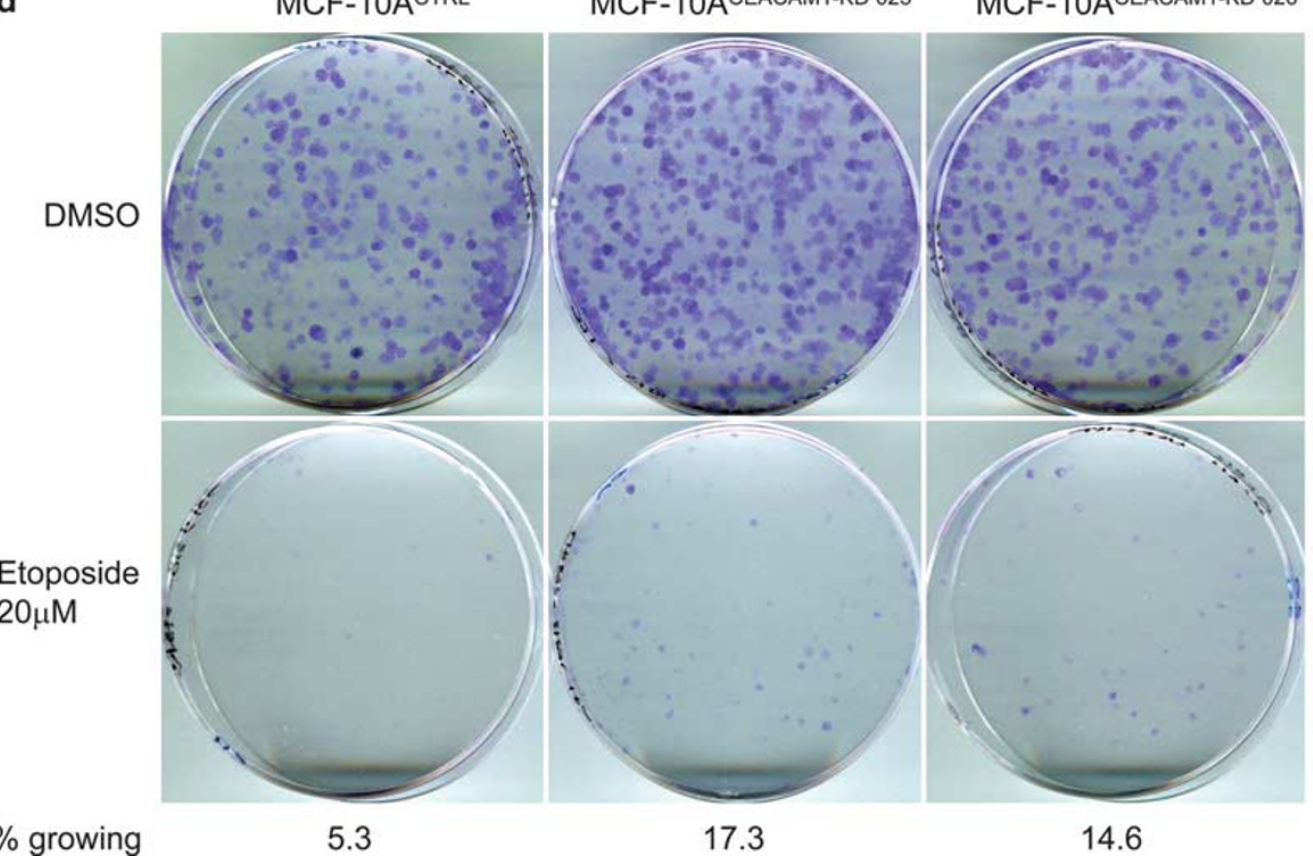


and MCF-10A ${ }^{\text {CEACAM1-KD } 026}$ cells than in MCF-10A ${ }^{\text {CTRL }}$ cells, relative to the respective untreated cultures. Similar results were obtained in proliferation assays using six-well plates, with a cell density of 5000 cells/well (data not shown). Similar results were obtained with HCT116 CEACAM1-KD 026, HCT116 CEACAM1-KD 028 or HCT116 ${ }^{\text {CTRL }}$ cells (1000 cells/60 mm Petri dish) (Figure 3d and Supplementary Table SI). By Annexin V labeling, little or no differences in apoptosis were observed between HCT116 stable transfectants 4 days after treatment with $20 \mu \mathrm{m}$ etoposide (data not shown). Morphological assessment of MCF-10A CEACAM1-KD 025 MCF-10A ${ }^{\text {CEACAM 1-KD } 026}$ or MCF-10A ${ }^{\text {CTRL }}$ cells treated with NCS for 6 days showed that the large majority of MCF-10A CTRL cells treated with NCS were morphologically senescent, whereas MCF-10A ${ }^{\text {CEACAM1-KD } 025}$ and MCF-10A CEACAM1-KD 026 cells treated with NCS displayed a significantly higher number of cells with normal morphology that regrew after the initial growth arrest (Figure 4a). The 5-ethynyl-2'-deoxyuridine (EdU) incorporation revealed a higher fraction of labeled cells in HCT116 ${ }^{\text {CEACAM1-KD } 026}$ cells compared with HCT116 $6^{\mathrm{CTRL}}$ cells 4 days after treatment with $20 \mu \mathrm{m}$ etoposide (the ratio of EdU labeled cells between etoposide vs dimethyl sulphoxide treated cells was $0.34 \pm 0.1$ for $\mathrm{HCT} 116^{\mathrm{CTRL}}$ cells and $0.57 \pm 0.1$ for HCT1 16 ${ }^{\text {CEACAM1-KD } 026}$ cells; errors indicate s.d., $n=2$ ). Quantification of senescence associated $\beta$-galactosidase staining revealed a lower number of senescent cells in MCF-10A CEACAM1-KD 025 and MCF-10A CEACAM1-KD 026 cells treated with etoposide compared with MCF-10A ${ }^{\text {CTRL }}$ cells 4 days after treatment (Figures $4 b$ and $c$ ).

As the $p 21 / p R b$ signaling pathway is a master regulator of growth arrest and senescence, we examined the p21/pRb status in MCF-10A cells with or without stable CEACAM1 silencing at the time of regrowth (3-5 days after NCS or etoposide treatment). We found that MCF-10A ${ }^{\text {CEACAM1-KD } 025}$ and MCF-10A ${ }^{\text {CTRL }}$ cells have a comparable $\mathrm{p} 53 / \mathrm{p} 21 / \mathrm{pRb}$ phosphorylation status or p21 mRNA or protein levels in response to NCS or etoposide (Figures $5 a$ and $b$ and data not shown). Similar results were obtained with HCT116 CEACAM1 - KD 026, HCT116 CEACAM1-KD 028 or HCT116 ${ }^{\text {CTRL }}$ cells after treatment with $20 \mu \mathrm{m}$ etoposide (Figure $5 \mathrm{c}$ ). a
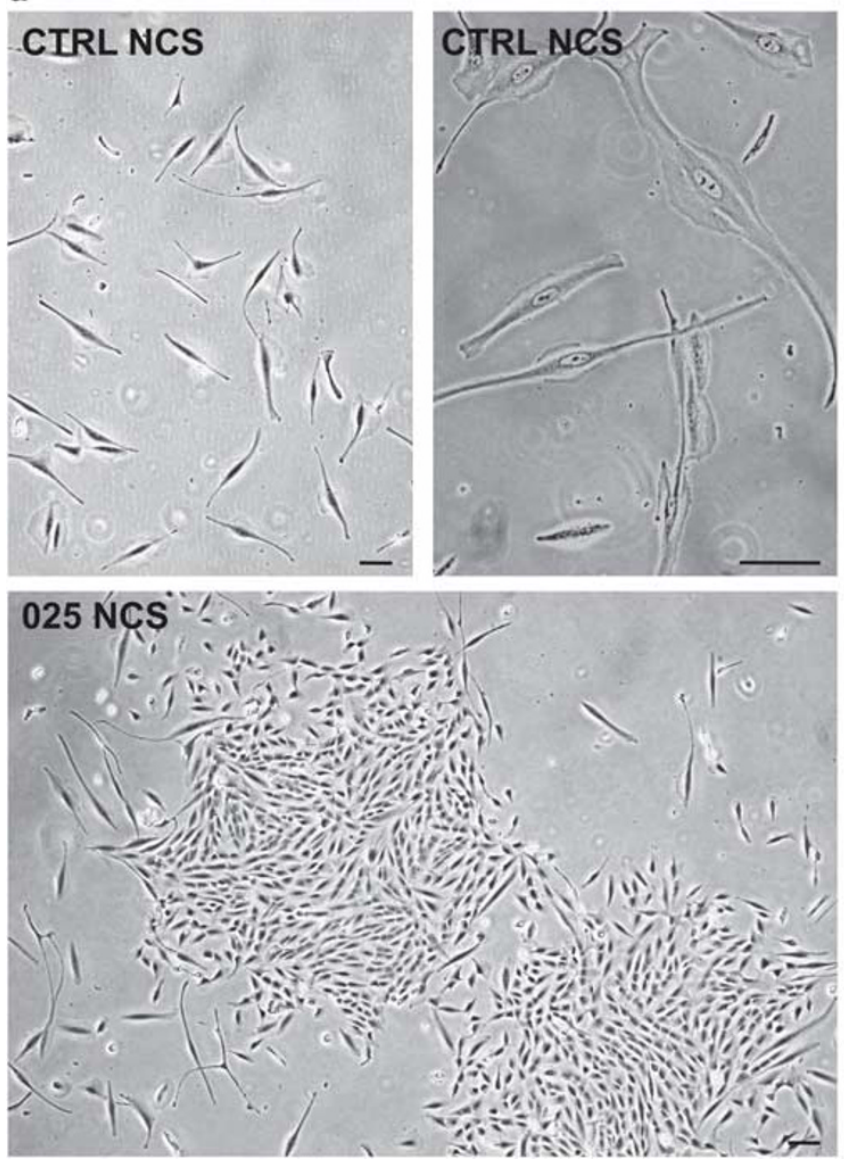

b

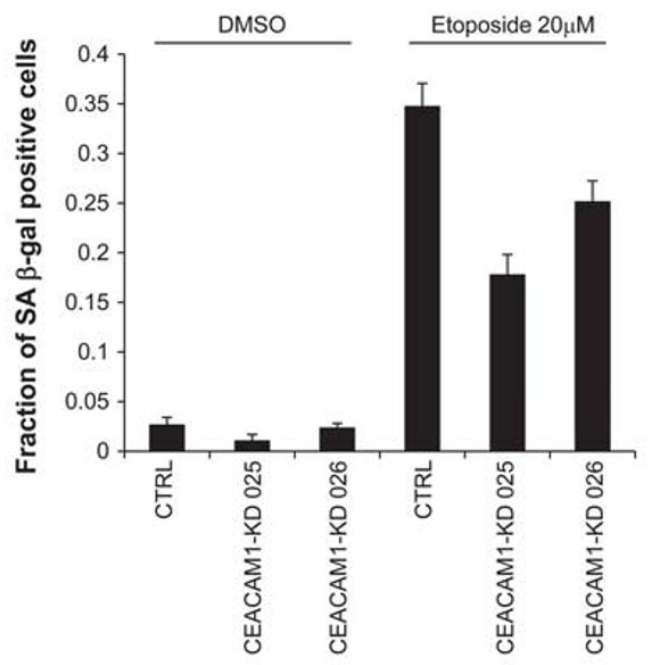

c

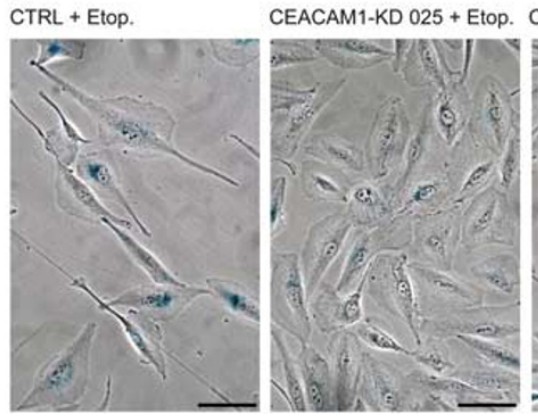

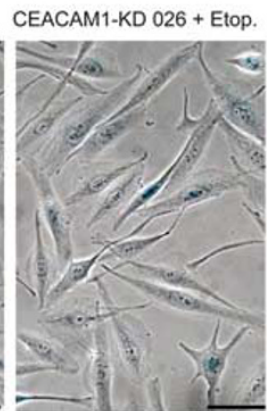

Figure 4. CEACAM1 is required for the establishment of cellular senescence as assessed by cellular morphology and senescence-associated $\beta$-galactosidase staining. (a) MCF-10A cells stably transfected with CEACAM1 shRNA vector no. 25 (025) or with a control shRNA vector (CTRL) were seeded in $60 \mathrm{~mm}$ Petri dishes at the density of 16000 cells/dish. On the following day the cells were treated with NCS $1.47 \mathrm{nM}$. After 6 days, the cells were photographed under phase contrast. Bar $=100 \mu \mathrm{m}$. (b) MCF-10A cells stably transfected with CEACAM1 shRNA vector no. 25 (CEACAM1-KD 025), with CEACAM1 shRNA vector no. 26 (CEACAM1-KD 026) or with a control shRNA vector (CTRL) were seeded in 6-well plates at the density of 50000 cells/well in triplicates. On the following day the cells were treated with the indicated dose of etoposide for $1 \mathrm{~h}$, washed with phosphate-buffered saline, and allowed to grow in new medium at $37^{\circ} \mathrm{C}$. After 4 days, the cells were stained for senescence-associated (SA) $\beta$-galactosidase. The graph represents the fraction of SA $\beta$-galactosidase positive cells \pm s.e.m. in 15 randomly selected photographic fields where a total of at least 250 cells from two different experiments/condition were counted. $p$ CEACAM1-KD $025+$ etoposide vs CTRL + etoposide $<0.001 ; p$ CEACAM1-KD $026+$ etoposide vs CTRL + etoposide $=0.005$. Two-sided $t$-test. (c) Examples of the (SA) $\beta$-galactosidase staining of MCF-10A ${ }^{\text {CTRL }}$, MCF-10A ${ }^{\text {CEACAM1-KD } 025}$, or MCF-10A CEACAMP-KD 026 cells treated with Etoposide as detailed and quantified in b. Bar $=100 \mu \mathrm{m}$. 


\section{DISCUSSION}

There is increasing evidence that cellular senescence is an important barrier to cancer development. In early tumor lesions, the dysregulated activity of cellular oncogenes leads to apoptosis or cellular senescence, and this is believed to occur as a consequence of DSBs resulting from oncogene-induced proliferation stress. The ATM/p53 pathway has a key role in these responses, and it has been suggested that the loss of components of this pathway in senescent preneoplastic lesions is causally involved in tumor progression. ${ }^{5-7}$ However, little is known about the physiological mediators of cellular senescence downstream of ATM/p53.

In this study, we report that CEACAM1, an immunoglobulin superfamily member whose expression is frequently lost in human tumors and which exhibits features of a tumor suppressor in several experimental systems, is consistently and strongly upregulated in response to several different DSB inducers in many different types of human cells, that this occurs starting from the lowest doses of DSB inducers, and that CEACAM1 induction by DNA damage is required for the establishment of cellular senescence in MCF-10A human mammary epithelial cells and HCT116 human colon cancer cells, the latter being a wellestablished and intensely studied model of p53 function. Given the amplitude of CEACAM1 induction by DNA damage and the fact that we observed this regulatory effect in all the ATM and p53 competent cell types analyzed, we were surprised by the fact that it has not been reported previously despite intensive investigations of p53-mediated transcriptional responses during the last two decades. Potential explanations include the particularly high amplitude of CEACAM1 induction by DNA damage in the cell line used for the initial CDNA microarray (MCF-10A), the slower induction of CEACAM1 by DNA damage compared with other p53-regulated genes (see Figure 1d) and the complete lack of expression of CEACAM1 in fibroblasts, frequently used for the analysis of p53 transcriptional responses, either under normal culture conditions or after treatment with DSB inducers (our unpublished results).

In our experiments, following treatment with etoposide or NCS, MCF-10A cells with stable CEACAM1 silencing initially arrest their proliferation similarly to controls with normal CEACAM1 expression, indicating that silencing of CEACAM1 does not cause defects in the main cell cycle checkpoints within the first $24 \mathrm{~h}$ after DNA damage. This observation is consistent with the slower induction of CEACAM1 by DNA damage compared with other p53-regulated genes, in particular CDKN1A/p21 (Figure 1d), and with the observation that the $\mathrm{p} 21 / \mathrm{pRb}$ axis appears to be regulated normally in MCF-10A cells and HCT116 cells with stable CEACAM1 silencing. Therefore, in terms of cell cycle arrest and senescence, the DNA damage response appears to generate at least two successive types of signals. The first type is necessary for initial cell growth arrest. This arrest is reversible, known to rely on $\mathrm{p} 21 / \mathrm{pRb}$ as its main regulator, and independent of CEACAM1. The second type of signals may include the induction of senescence genes (including CEACAM1) and might actually be operating to promote senescence under certain conditions, for example if the initial arrest persists. In this context, however, normal regulation of the p21/Rb axis by DNA damage does not appear to be sufficient for the establishment of cellular senescence, as clearly indicated by the observation that $\mathrm{p} 21 / \mathrm{Rb}$ regulation appears to occur normally in MCF-10A and HCT116 cells with stable CEACAM1 silencing 3-5 days after DNA damage, when regrowth is observed. Similar results have recently been reported in other cell culture systems

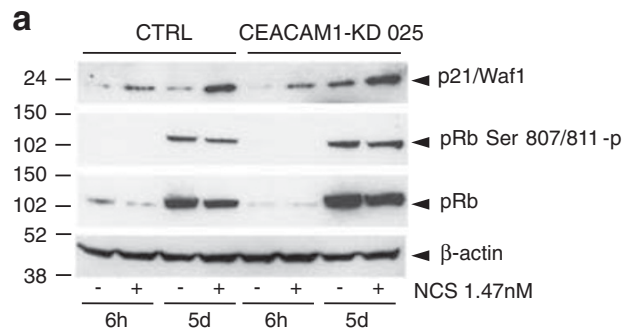

b

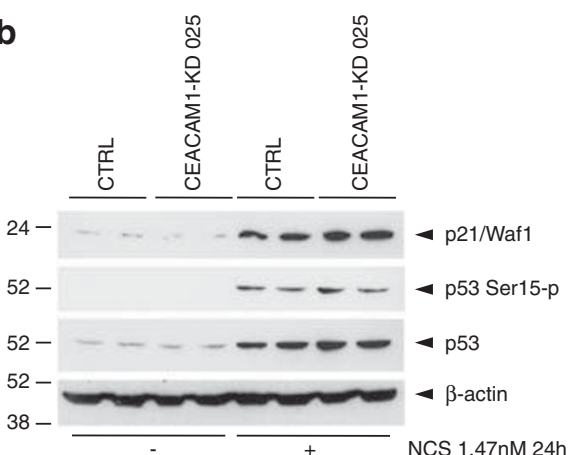

C

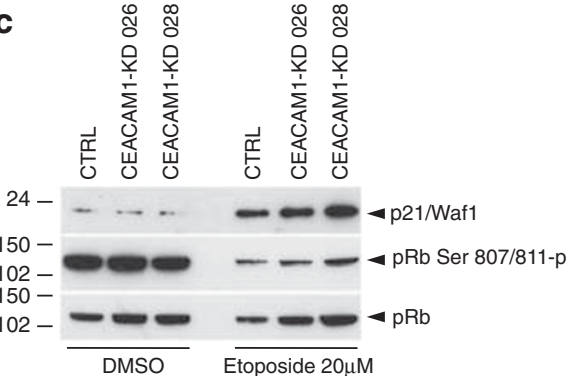

Figure 5. MCF-10A and HCT116 cells with stable silencing of CEACAM1 exhibit normal regulation of p21/pRb in response to DNA damage. (a) MCF-10A cells stably transfected with CEACAM1 shRNA vector no. 25 (CEACAM1-KD 025) or with a control shRNA vector (CTRL) were incubated in the presence or the absence of $1.47 \mathrm{nM}$ NCS for the indicated time points, lysed, and analyzed for p21/Waf1, pRb Ser 807/811-p, $\mathrm{pRb}$, or $\beta$-actin by western blotting. (b) MCF-10A cells stably transfected with CEACAM1 shRNA vector no. 25 (CEACAM1-KD 025) or with a control shRNA vector (CTRL) were incubated in the presence or the absence of NCS $1.47 \mathrm{nM}$ for $24 \mathrm{~h}$ in duplicate as indicated, lysed, and analyzed for $\mathrm{p} 21 /$ Waf1, pSer15-p, p53, or $\beta$-actin by western blotting. (c) HCT116 cells stably transfected with CEACAM1 shRNA vector no. 26 (CEACAM1-KD 026), with CEACAM1 shRNA vector no. 28 (CEACAM1-KD 028) or with a control shRNA vector (CTRL) were incubated in the presence of $20 \mu \mathrm{m}$ etoposide or dimethyl sulfoxide (DMSO (solvent)) for $1 \mathrm{~h}$, washed with phosphate-buffered saline and incubated in new medium for 3 days. At the end of the incubation the cells were lysed and analyzed for p21/Waf1, pRb Ser 807/811-p or pRb by western blotting. 
for two other p53-regulated, senescence-inducing genes, namely plasminogen activator inhibitor- $1(\mathrm{PAl}-1)^{17}$ and DEC1/Stra13, ${ }^{18}$ thus supporting the idea that, in addition to $\mathrm{p} 21 / \mathrm{Rb}$, the establishment of cellular senescence requires other molecules. However, in contrast to CEACAM1, PAI-1 has no reported tumorsuppressor features, and experimental evidence that DEC1/Stra13 is a tumor suppressor is limited, as although DEC1/Stra13 overexpression inhibits the growth of esophageal squamous cell carcinoma cell lines, ${ }^{19}$ no cancer phenotype has been reported in DEC1/Stra13 knockout mice. ${ }^{20,21}$

Several different $\mathrm{p} 53$ dependent or independent genes regulate senescence in tissue culture. Given the importance of cellular senescence in limiting cellular proliferation, this might reflect redundancy of the genes regulating this cellular function to ensure its execution when needed, or cell type specific requirements for signaling pathways leading to senescence. It is also possible that some of the senescence inducing genes are hierarchically more important than others, thus resulting in a tumor-suppressor activity in several experimental systems as reported for CEACAM1. Whatever the correct interpretation for this apparent functional redundancy might be, to our knowledge CEACAM1 is the first known p53-regulated tumor-suppressor gene inducing cellular senescence in response to DNA damage in cell culture, and therefore the first described bona fide physiological mediator of cellular senescence downstream of p53. However, further studies are necessary to assess whether CEACAM1 actually suppresses tumor growth in vivo by inducing senescence downstream of p53. For example, it would be interesting to investigate whether increased colon carcinogenesis in CEACAM1 knockout mice treated with azoxymethane ${ }^{22}$ correlates with deficient induction of cellular senescence in colon epithelial cells.

In our view, two additional points deserve consideration. First, very little data is currently available on CEACAM1 mutations in human cancer. As an ATM and p53-regulated gene, CEACAM1 is a potential target for mutations in cancer. Such mutations, if any, might account in part for the observed protein loss in human tumor tissue samples, or protein overexpression in cancers where CEACAM1 overexpression occurs. If CEACAM1 mutants exist in cancer, it would be very interesting to study both the distribution pattern of such mutants with respect to $\mathrm{p} 53$ status, and potential defects of their activity in tissue culture and transgenic mice, in particular in the setting of the DNA damage response.

Second, the mechanism by which CEACAM1 regulates senescence deserves investigation. CEACAM1 is known to have a generally negative regulatory effect on cell proliferation, an effect mediated in part by negative CEACAM1 regulation of the insulin receptor and epidermal growth factor receptor signaling. ${ }^{1}$ Although it is possible that this activity contributes to senescence by delaying or arresting cell proliferation in some experimental settings, the role of CEACAM1 in senescence needs to be investigated in the context of the transcriptional changes occurring during the DNA damage response. A first step will be the study of the role of CEACAM1 in the regulation of other genes involved in cellular senescence.

\section{MATERIALS AND METHODS}

\section{Cell culture}

MCF-10A cells were bought from American type culture collection and cultured as described. ${ }^{3}$ MCF-10A cells with stable ATM silencing were reported previously. ${ }^{3}$ HCT116 and SW48 colon cancer cells (and p53 -/derivatives) were kindly provided by $\operatorname{Dr} B$ Vogelstein. The cells were grown in McCoy's 5A Medium (cat. no. M8403, Sigma, Buchs, Switzerland) supplemented with $10 \%$ heat-inactivated fetal calf serum (cat no. 2-01F30-I, Amimed/Bioconcept, Allschwil, Switzerland), L-Glutamine, and PenicillinStreptomycin (Sigma P0781). Neocarzinostatin was prepared and purified as described. ${ }^{23}$ Etoposide (E1383) and nutlin-3 (N6287) were purchased from
Sigma. The ATM inhibitor 2-morpholin-4-yl-6-thianthren-1-yl-pyran-4-one (KU-55933) was purchased from Calbiochem (Darmstadt, Germany, cat. no. 118500).

Quantitative real-time polymerase chain reaction

cDNA was synthesized from $1 \mu \mathrm{g}$ of total RNA using random hexamers and Superscript III reverse transcriptase (Invitrogen) according to the manufacturer's instructions. SYBR Green assays were designed using the program Primer Express v 2.0 (Applied Biosystems, Rotkreuz, Switzerland) with default parameters. Amplicon sequences were aligned against the human genome by BLAST to ensure that they were specific for the gene being tested. Oligonucleotides were purchased from Invitrogen. The efficiency of each design was tested with serial dilutions of CDNA. PCRs (10 $\mu$ l volume) contained diluted CDNA, $2 \times$ Power SYBR Green Master Mix (Applied Biosystems), and $300 \mathrm{~nm}$ forward and reverse primers. PCR were performed on a SDS $7900 \mathrm{HT}$ instrument (Applied Biosystems) with the following parameters: $50{ }^{\circ} \mathrm{C}$ for $2 \mathrm{~min}, 95^{\circ} \mathrm{C}$ for $10 \mathrm{~min}$, and 45 cycles of $95^{\circ} \mathrm{C}$ for $15 \mathrm{~s}$ and $60^{\circ} \mathrm{C}$ for $1 \mathrm{~min}$. Each reaction was performed in three replicates on a 384-well plate. Raw $C t$ values obtained with SDS version 2.2 (Applied Biosystems) were imported in Excel, and normalization factor and fold changes were calculated using the GeNorm method. ${ }^{24}$ Oligonucleotide primer sequences were as follows:

ATM: $5^{\prime}$-TGCTGACAATCATCACCAAGTTC-3', 5'-TCTCCCTTCGTGTCCTGGAA-3'; ATF3: $5^{\prime}$-TGCAAAGTGCCGAAACAAGA-3', 5'-GCATTCACACTTTCCAGCTTCTC-3' BIRC3: 5'-AGAGAGAAAGAGCAACTGAGGAAAAA-3', 5'-TGCCATTCTATTCTTCC GGATTA-3';

BTG2: 5'-GCTCCAGGAGGCACTCACA-3', 5'-AGCCCTTGGACGGCTITT-3'; CEACAM1: 5'-CGACCCCATCATGCTGAAC-3', 5'-CAATGGCCCCAGGTGAGA-3'; CEACAM1-L: 5'-CAAGCGACCAGCGTGATCT-3', 5'-TGGTCCTGAGTGTGGTT GGA-3';

CEACAM1-S: $5^{\prime}$-CCCTGGCATGTTTTCTGCA-3', $5^{\prime}$ - TGGAGTGGTCCTGAGCTGC-3'; CEACAM1 int-ex: 5'-CCCTAATGCATAGGTCCCAATATT-3', 5'-CGGGTTCC AGAAGGTTAGAAGTG-3';

IRF1: 5'-CATGGCTGGGACATCAACAAG-3', 5'-CTGCTTTGTATCGGCCTGTGT-3'; MDM2: 5'-TCTACAGGGACGCCATCGA-3', 5'-CTGATCCAACCAATCACCTGAA-3'; RELB: 5'-TTTCCGAGCCCGTCTATGAC-3', 5'-TCCGCAGCTCTGATGTGTT- $3^{\prime}$; p21: 5'-TCAGAGGAGGCGCCATGT-3', 5'-TGTCCACTGGGCCGAAGA-3';

p53: 5'-CAAAGAAGAAACCACTGGATGGA-3', 5'-CAGCTCTCGGAACATC TCGAA-3'.

CEACAM1 primers amplify CEACAM1 mature mRNA between base positions 1356 and 1427 (ENST00000161559). CEACAM1 int-ex primers amplify CEACAM1 primary transcript between base positions 861 and 937 (ENST00000161559) at the intron/exon two boundary (http://www.ensembl.org/Homo_sapiens/Transcript/Sequence_cDNA?g=ENS G00000079385;r=19:43011458-43032661;t=ENST00000161559).

\section{Western blotting}

Cells were lysed using radioimmune precipitation assay buffer (cat. no. R0278, Sigma) and sonication for total protein extraction. For pRb analysis, cells were lysed in Tris- $\mathrm{HCl} 62.5 \mathrm{~mm} \mathrm{pH} 6.8$, sodium dodecyl sulfate $2 \%$, sonicated, and heated at $95^{\circ} \mathrm{C}$ for $5 \mathrm{~min}$. In either cases, Halt protease inhibitor mixture (cat. no. 78410, Pierce/Thermo Fisher Scientific, Lausanne, Switzerland) and phosphatase inhibitor mixture (cat. no. 78420, Pierce/ Thermo Fisher Scientific) were added to the lysis buffer. Fifty to eighty $\mu \mathrm{g}$ of protein was run in sodium dodecyl sulfate-polyacrylamide gels and transferred to nitrocellulose membranes (cat. no. RPN 303 D, GE Healthcare, Glattbrugg, Switzerland). Blots were incubated with antibodies against CEACAM1 (cat. no. H00000634-B01P, Abnova/Labforce, Nunningen, Switzerland), ATM (cat. no. ab78, Abcam plc, Cambridge, United Kingdom), ATM Ser(P)-1981 (cat. no. 4526, Cell Signaling Technology/Bioconcept, Allschwil, Switzerland), Nijmegen breakage syndrome (NBS)-1 (cat. no GTX70224, GeneTex, Irvine, CA, USA), p53 (cat. no. 628201, Biolegend/ Lucerna-Chem, Luzern, Switzerland), p53 Ser(P)-15 (cat. no. PC386, Merck, Darmstadt, Germany), p21 WAF1/CIP1 (cat. no. M7202, Dako, Baar, Switzerland), Phospho-Rb Ser(P)-807/811 (cat. no. 9308, Cell Signaling Technology), Rb (cat. no. 9309, Cell Signaling Technology) or $\beta$-actin (cat. no ab6276, Abcam plc), according to the manufacturer's instructions. Horse- 
radish peroxidase-conjugated secondary antibody-primary antibody complexes were revealed on the nitrocellulose membranes using the LumiLight (cat. no. 2015200, Roche Applied Science, Rotkreuz, Switzerland) or Lumi-Light Plus (cat. no. 12015196001, Roche Applied Science) western blotting substrate. Scanning densitometry was performed using a Bio-Rad Universal Hood II instrument and Quantity One (version 4.5.2) acquisition software (Bio-Rad, Reinach, Switzerland).

\section{siRNA transfection}

MCF-10A cells were transfected with p53 siRNA no. 3 (target sequence: $5^{\prime}$-CA GAGTGCATTGTGAGGGTTA-3'; Qiagen cat. no. SI00011655, Qiagen, Hombrechtikon, Switzerland), no. 9 (target sequence: 5'-AAGGAAATTGCGTGTGGAGT-3'; Qiagen cat. no. SI02655170) or control siRNA (Qiagen proprietary sequence; Qiagen cat. no. SI03650318) using HiPerFect transfection reagent (Qiagen cat. no. 301702) according to the manufacturer's instructions.

\section{Generation of MCF-10A and HCT116 stable transfectants with CEACAM1 silencing}

MCF-10A and HCT116 stable transfectants with stable CEACAM1 silencing were established using shRNA constructs against CEACAM1 in pRS vector purchased from OriGene Technologies/Labforce, Nunningen, Switzerland (cat. no. TR314008), according to the manufacturer's instructions. Target sequences in CEACAM1 were as follows:

shRNA 025: 5'-GACACTGGAATCTCCATCCGTTGGTTCTT-3'; shRNA 026: 5'-GGAAAGAGTGGATGGCAACCGTCAAATTG-3'; shRNA 028: 5'-CACTCTACTCAGTGTCACAAGGAATGACA-3'.

\section{Proliferation assays}

Cells were seeded in $60 \mathrm{~mm}$ Petri dishes at the cell density of 1000 cells/ dish (HCT116 transfectants) or 1500 cells/dish (MCF-10A transfectants). On the following day, the cells were treated with the indicated doses of etoposide ( $1 \mathrm{~h}$, followed by phosphate-buffered saline wash and medium change) or NCS and allowed to grow for 7 days thereafter. Colonies were fixed with methanol, stained with Crystal Violet, and counted.

Senescence-associated $\beta$-galactosidase staining was performed with the SA $\beta$-gal staining kit (Cell Signaling cat. no. 9860) according to the manufacturer's instructions.

\section{CONFLICT OF INTEREST}

The authors declare no conflict of interest.

\section{ACKNOWLEDGEMENTS}

We thank B Foglia and C Barraclough for the skillful technical assistance; Dr P Descombes for advice in real-time quantitative PCR; Professor T Halazonetis and $F$ Toledo for critical reading of the manuscript; $\operatorname{Dr} B$ Vogelstein for kindly providing the HCT116 and SW48 cells; Drs B Vogelstein and A Levine for kindly replying to all our questions on p53 by email, and Professors D Belin and J-D Vassalli for their support. This work was supported by grants from the Ligue Genevoise Contre le Cancer, from the Fondation pour la Lutte contre le Cancer et pour des Recherches Médico-Biologiques, and from the Fondation Ritva et Pierre Meyer.

Author contributions: APS: conception and writing. RB, QS, MF, LL, FGP: experiments and data analysis. WR, VF: experiment planning, data analysis, supervision and writing. SJM: conception, experiment planning, data analysis, supervision and writing.

\section{REFERENCES}

1 Kuespert K, Pils S, Hauck CR. CEACAMs: their role in physiology and pathophysiology. Curr Opin Cell Biol 2006; 18: 565-571.

2 Nouvion AL, Beauchemin N. CEACAM1 as a central modulator of metabolism, tumor progression, angiogenesis and immunity]. Med Sci 2009; 25: 247-252.
3 Mandriota SJ, Buser R, Lesne L, Stouder C, Favaudon V, Maechler P. et al. Ataxia telangiectasia mutated (ATM) inhibition transforms human mammary gland epithelial cells. J Biol Chem 2010; 285: $13092-13106$.

4 Renwick A, Thompson D, Seal S, Kelly P, Chagtai T, Ahmed M et al. ATM mutations that cause ataxia-telangiectasia are breast cancer susceptibility alleles. Nat Genet 2006; 38: 873-875.

5 Gorgoulis VG, Halazonetis TD. Oncogene-induced senescence: the bright and dark side of the response. Curr Opin Cell Biol 2010; 22: 816-827.

6 Collado M, Serrano M. Senescence in tumours: evidence from mice and humans. Nat Rev Cancer 2010; 10: $51-57$.

7 Evan Gl, d'Adda di Fagagna F. Cellular senescence: hot or what? Curr Opin Genet Dev 2009; 19: 25-31.

8 Hickson I, Zhao Y, Richardson CJ, Green SJ, Martin NM, Orr Al et al. Identification and characterization of a novel and specific inhibitor of the ataxia-telangiectasia mutated kinase ATM. Cancer Research 2004; 64: 9152-9159.

9 Rashi-Elkeles S, Elkon R, Weizman N, Linhart C, Amariglio N, Sternberg G et al. Parallel induction of ATM-dependent pro- and antiapoptotic signals in response to ionizing radiation in murine lymphoid tissue. Oncogene 2006; 25: $1584-1592$.

10 Elkon R, Rashi-Elkeles S, Lerenthal Y, Linhart C, Tenne T, Amariglio N et al. Dissection of a DNA-damage-induced transcriptional network using a combination of microarrays, RNA interference and computational promoter analysis. Genome Biol 2005; 6: R43.

11 Bunz F, Dutriaux A, Lengauer C, Waldman T, Zhou S, Brown JP et al. Requirement for p53 and p21 to sustain G2 arrest after DNA damage. Science 1998; 282: $1497-1501$.

12 Fusenig NE, Boukamp P. Multiple stages and genetic alterations in immortalization, malignant transformation, and tumor progression of human skin keratinocytes. Mol carcinog 1998; 23: 144-158.

13 Bartek J, Iggo R, Gannon J, Lane DP. Genetic and immunochemical analysis of mutant p53 in human breast cancer cell lines. Oncogene 1990; 5: 893-899.

14 Vassilev LT, Vu BT, Graves B, Carvajal D, Podlaski F, Filipovic Z et al. In vivo activation of the p53 pathway by small-molecule antagonists of MDM2. Science 2004; 303: $844-848$.

15 Hoh J, Jin S, Parrado T, Edington J, Levine AJ, Ott J. The p53MH algorithm and its application in detecting p53-responsive genes. Proc Natl Acad Sci USA 2002; 99: 8467-8472.

16 Gencheva M, Chen CJ, Nguyen T, Shively JE. Regulation of CEACAM1 transcription in human breast epithelial cells. BMC Mol Biol 2010; 11: 79.

17 Kortlever RM, Higgins PJ, Bernards R. Plasminogen activator inhibitor-1 is a critical downstream target of p53 in the induction of replicative senescence. Nat Cell Biol 2006; 8: 877-884

18 Qian Y, Zhang J, Yan B, Chen X. DEC1, a basic helix-loop-helix transcription factor and a novel target gene of the $\mathrm{p} 53$ family, mediates $\mathrm{p} 53$-dependent premature senescence. J Biol Chem 2008; 283: 2896-2905.

19 Yang L, Leung AC, Ko JM, Lo PH, Tang JC, Srivastava G et al. Tumor suppressive role of a $2.4 \mathrm{Mb} 9 \mathrm{q} 33-\mathrm{q} 34$ critical region and DEC1 in esophageal squamous cell carcinoma. Oncogene 2005; 24: 697-705.

20 Sun H, Lu B, Li RQ, Flavell RA, Taneja R. Defective T cell activation and autoimmune disorder in Stra13-deficient mice. Nat Immunol 2001; 2: 1040-1047.

21 Seimiya M, Wada A, Kawamura K, Sakamoto A, Ohkubo Y, Okada S et al. Impaired lymphocyte development and function in Clast5/Stra13/DEC1-transgenic mice. Eur J Immunol 2004; 34: 1322-1332.

22 Leung N, Turbide C, Olson M, Marcus V, Jothy S, Beauchemin N. Deletion of the carcinoembryonic antigen-related cell adhesion molecule 1 (Ceacam1) gene contributes to colon tumor progression in a murine model of carcinogenesis. Oncogene 2006; 25: 5527-5536.

23 Favaudon V. Gamma-radiolysis study of the reductive activation of neocarzinostatin by the carboxyl radical. Biochimie 1983; 65: 593-607.

24 Vandesompele J, De Preter K, Pattyn F, Poppe B, Van Roy N, De Paepe A et al. Accurate normalization of real-time quantitative RT-PCR data by geometric averaging of multiple internal control genes. Gen Biol 2002; 3: RESEARCH0034.

\section{(c)}

Oncogenesis is an open access journal published by Nature Publishing Group. This work is licensed under the Creative Commons AttributionNonCommercial-No Derivative Works 3.0 Unported License. To view a copy of this license, visit http://creativecommons.org/licenses/by-nc-nd/3.0/

Supplementary Information accompanies the paper on the Oncogenesis website (http://www.nature.com/oncsis) 